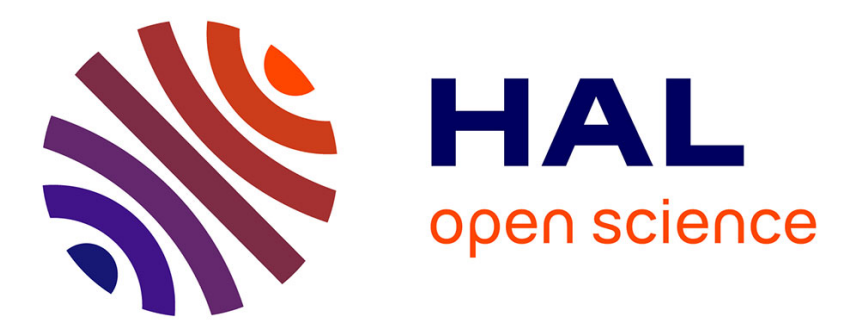

\title{
Acoustic methods for measuring the porosities of porous materials incorporating dead-end pores
}

\author{
Thomas Dupont, Philippe Leclaire, Raymond Panneton
}

\section{To cite this version:}

Thomas Dupont, Philippe Leclaire, Raymond Panneton. Acoustic methods for measuring the porosities of porous materials incorporating dead-end pores. Journal of the Acoustical Society of America, 2013, 133 (4), pp.2136-2145. 10.1121/1.4792484 . hal-01001818

\section{HAL Id: hal-01001818 https://hal.science/hal-01001818}

Submitted on 5 Jun 2014

HAL is a multi-disciplinary open access archive for the deposit and dissemination of scientific research documents, whether they are published or not. The documents may come from teaching and research institutions in France or abroad, or from public or private research centers.
L'archive ouverte pluridisciplinaire HAL, est destinée au dépôt et à la diffusion de documents scientifiques de niveau recherche, publiés ou non, émanant des établissements d'enseignement et de recherche français ou étrangers, des laboratoires publics ou privés. 


\title{
Acoustic methods for measuring the porosities of porous materials incorporating dead-end pores
}

\author{
Thomas Dupont and Philippe Leclaire \\ Département de Recherche en Ingénierie des Véhicules pour l'Environnement, Institut Supérieur de l'Automobile et des Transports, \\ Université de Bourgogne, 49 Rue Mademoiselle Bourgeois, 58027 Nevers Cedex, France
}

Raymond Panneton

Groupe d' Acoustique de l' Université de Sherbrooke, Department of Mechanical Engineering, Université de Sherbrooke, Sherbrooke, Québec JIK 2Rl, Canada

The acoustic properties of porous materials containing dead-end (DE) pores have been proposed by Dupont [J. Appl. Phys. 110, 094903 (2011)]. In the theoretical description, two physical parameters were defined (the dead-end porosity and the average length of the dead-end pores). With the knowledge of the open porosity (measured with non-acoustic methods), and the measurement of ki-nematic porosity (also called the Biot porosity in this article), it is possible to deduce the dead-end porosity. Two acoustic methods for measuring the Biot porosity for a wide range of porosities are proposed. These methods are based on acoustic transmission and on the low and high frequency behaviors of acoustic indicators. The low frequency method is valid for high porosities. It involves measurements in a transmission tube and the knowledge of the theoretical asymptotic behavior of the phase velocity at high frequencies. The high frequency method is based on ultrasonic measurements and on the high frequency asymptotic behavior of the transmission coefficient. It is well adapted for material with relatively low values of porosity. Good precision was found for both methods and materials containing dead end porosity were tested.

\section{INTRODUCTION}

The porosity most often considered in the acoustics of porous media is open porosity. However, different kinds of porosities can be defined in porous materials, ${ }^{1,2}$ depending on the microstructure (pore geometry, interconnections, possible existence of inclusions). These include the following: Total porosity, closed porosity, open porosity, effective or kinematic porosity, and dead-end porosity. The total porosity $\phi_{T}$ corresponds to the total volume of fluid contained in the material. It is given by the sum of the open porosity $\phi_{O}$ and of the closed porosity $\phi_{C}$ :

$$
\phi_{T}=\phi_{O}+\phi_{C} .
$$

The open porosity is defined as the fraction of interconnected air volume to the total volume of the sample. It encompasses the kinematic $\phi_{B}$ and the dead-end $\phi_{D E}$ porosities:

$$
\phi_{O}=\phi_{B}+\phi_{\mathrm{DE}}
$$

The kinematic porosity $\phi_{B}$ (also called the effective porosity) is defined as the ratio of the "mobile volume" of saturation fluid released under the effect of a complete drainage to the bulk volume of the porous material. The kinematic porosity is the one used in the Biot acoustic model and will therefore be referred to as the "Biot porosity" for the remainder of this paper.
The dead-end (DE) porosity $\phi_{D E}$ is defined by the ratio of "non-moving fluid volume" (this vol-ume is included in the volume of interconnected pores) to the bulk volume of the porous material. $\phi_{D E}$ results from the fact that a cell, although connected to another cell at one end, can remain closed at the other end so that the fluid may not flow through it.

There has been a number of studies dealing with DE pores and their effect on the acoustic behavior of materials. Some of these materials (for example soils) have been studied in hydrogeology sciences for petroleum and gas prospection applications. ${ }^{2}$ Fatt $^{3}$ studied the influence of DE pores placed in a configuration on the relative permeability of materials in which two or more fluids may flow. In a neurobiological application on the brain, Hrabe et al. ${ }^{4}$ noted that DE pores and DE volumes could exist in extracellular space of the brain. They showed that the DE pores could alter the diffusion and increase the tortuosity. Chevillote et al. ${ }^{5}$ studied the sound-absorption properties of perforated closed-cell metallic foams. They observed that the DE pores created by the perforation process could have significant effects on acoustic behavior. We also think that new man-made structured materials such as meta-material may be viewed as materials containing DE pores. ${ }^{6,7}$

The acoustic properties of porous materials containing dead-end pores such as porous aluminum have recently been studied experimentally and theoretically by Dupont 
et $a l .{ }^{8}$ These kinds of materials can be obtained by dissolving salt grains embedded in a solid metal matrix with the help of water. ${ }^{9}$ The solid matrix is obtained after the metal in liquid form has invaded the granular material formed by the salt particles at negative pressure and high temperature and after cooling and solidification of the metal. The DE pores and their effects on acoustic behavior have been studied, and it has been found that, for these materials, the predictions of the classical models such as that of JohnsonChampoux-Allard (JCA model) were not as satisfying as for other materials. Indeed the fluid inside the DE pores could be acoustically inert and may not contribute to the attenuation by viscous friction of a passing wave. In addition, it is believed that, depending on the frequency, the fluid properties and the dimensions of the cavities, standing wave fields can settle within the dead-end pores, resulting in resonances in acoustic indicator curves. Initial confirmation of these predictions was provided by experimental results obtained on materials with cavities created artificially and on porous metals that are likely to contain deadend porosity. A new model based on the JCA model and the transfer matrix approach was proposed. ${ }^{1}$ Two new physical parameters were introduced, the dead-end porosity $\phi_{D E}$ and the average length of the dead-end pores $L_{D E}$. New material characterization techniques are being investigated to estimate these new parameters. The main objective of this paper is to propose two acoustic methods for measuring the DE porosity.

Two approaches for measuring porosities consist in nonacoustic and acoustics measurements. The non-acoustic methods are classical. They include different methods, such as the method based on Boyle's law for isothermal processes $\left(\right.$ Beranek $^{10}$ ), which was standardized [ASTM D2856-94 (Ref. 11)]. An improved method based on comparison of air volumes was proposed by Leclaire et al. ${ }^{12}$ Champoux et al. ${ }^{13}$ proposed an improved and modernized version of Beranek's method based on Boyle's law; Panneton and Gros ${ }^{14}$ proposed a technique based on missing mass; and Salissou and Panneton ${ }^{15}$ presented a method based on pressure/mass measurements. These classical methods provide measurements of the open porosity. However, they do not allow the distinction between the Biot and the DE porosities. Leclaire et al. ${ }^{16}$ proposed a method to determine the specific area of porous acoustic materials from water extraction data (from the integration of the water extraction curve). This method allows the measurement of the Biot porosity, but it is necessary to immerse the material in a heavy fluid like water. This can be a drawback because it may not be always possible to immerse a large panel of the material in water. Other methods exist that can provide measurements of the Biot porosity such as the tracer method, ${ }^{17}$ but they also have some disadvantages. Indeed these methods are not well adapted for a large panel of porous media: The tracer methods use water immersion with a flow through the medium. Moreover, these methods can produce significant experimental errors. Gibb et al. ${ }^{17}$ noted that the Biot porosity was measured equal to or greater than that calculated for total porosity. Tomography or microtomography methods ${ }^{18,19}$ can be used to determine the Biot porosity and the dead-end porosity. These methods can require expensive test benches.
Acoustic methods are based on the inversion of physical parameters from acoustic measurement data. Depending on the known parameters in the problem considered, the appropriate parameters can be deduced. Different acoustic methods were used in the past to determine the porosity, the tortuosity, the permeability, or the static airflow resistivity (the flow resistivity is inversely proportional to the permeability) or even the viscous and thermal characteristic lengths. In addition, low frequency methods (impedance tube measurements) and high frequency methods (ultrasonic measurement) can be distinguished. In the low frequency methods, ${ }^{20-22}$ impedance or transmission tube measurements are used, and an inversion procedure is implemented to deduce the appropriate parameters. The high frequency methods involve the use of ultrasonic waves in air-saturated materials. To estimate the porosity and the tortuosity, Fellah $e a^{23,24}$ proposed, in the time domain, a method based on the solution of inverse problem using the waves reflected by the first interface of porous material. This method requires measurements with two different incidence angles. Umnova et al..$^{25}$ proposed, in the time domain, a method to determine the tortuosity and the porosity from transmission and reflection on thick samples of porous material. Based on Panneton and Olny's approach ${ }^{20,21}$ for low frequencies, Groby ${ }^{26}$ proposed an analytical method in the frequency domain to estimate the JCA macroscopic parameters (including the porosity) with transmitted and reflected coefficients, at ultrasonic frequencies.

All the methods based on the reflection on the fluidporous medium interface yield the surface porosity and cannot be applied to materials with dead-end pores as the assumption of homogeneous and statistically isotropic material may not be fulfilled for dead-end porous materials. Fellah et al. ${ }^{24}$ noted that for weakly resistive porous media, it was possible to measure the wave reflected by the second interface of the sample, but in many cases, this reflected wave was strongly attenuated and the signal could not be exploited. Concerning the method based only on transmission (those susceptible to be used to determine the Biot porosity), Fellah et al. ${ }^{24}$ noted that the weak sensitivity of the transmission coefficient with porosity for media with values of tortuosity and porosity close to one created a problem for the determination of porosity. Thus no specific method based on ultrasonic transmission waves was proposed to measure the Biot porosity.

With a view to deduce the DE porosity $\phi_{D E}$, the objective of this paper is to measure the Biot porosity $\phi_{B}$ with acoustic methods for a large range of porosities and macroscopic parameters. We define a parameter given by the ratio of the Biot porosity and of the square root of tortuosity $\sqrt{\alpha_{\infty}}$ :

$$
\beta=\phi_{B} / \sqrt{\alpha_{\infty}} .
$$

The range of applicability of the two methods proposed in this paper will be determined by the parameter $\beta$. The motivation for defining this parameter will become apparent in the high frequency method.

Two simple acoustic transmission methods are proposed in this paper to estimate the Biot porosity and to deduce the DE porosity for a wide range of porous materials containing DE pores: A low frequency method using a transmission 
tube for material with $\beta$ close to one and a high frequency method using ultrasonic transducers, for materials with small values of $\beta$. Sections of this article were presented at a conference. ${ }^{27}$

\section{LOW FREQUENCY METHOD}

\section{A. Phase velocity and Biot's characteristic frequency}

This method is based on the theoretical asymptotic behavior at high frequencies (HF) of the phase velocity (or of the attenuation) used in combination with low frequency (LF) measurements (for example from an impedance tube). Biot's characteristic frequency separating the low and high frequency regimes (the viscous flow and inertial flow in the pore) is $f_{c}$. If $f<f_{c}$, the viscous forces dominate and if $f>f_{c}$ the inertial forces dominate. The Biot's characteristic frequency was defined by M. A. Biot, ${ }^{28}$ its expression is established with help of Ref. 29 and is given by ${ }^{20}$

$$
f_{c}=\sigma \frac{\phi_{B}}{2 \pi \alpha_{\infty} \rho_{f}},
$$

where $\sigma$ is the static air flow resistivity, $\alpha_{\infty}$ is the tortuosity and $\rho_{f}$ volume mass of the fluid. It can be noted that $f_{c}$ depends linearly on the Biot porosity. The phase velocity is given by (Chap. 1, p. 11 of Ref. 30)

$$
v_{p}=\frac{\omega}{\operatorname{Re}\left(k_{e q}\right)},
$$

where $\omega$ is the angular frequency and $\operatorname{Re}\left(k_{e q}\right)$ is the real part of the equivalent wave number. The HF asymptote of phase velocity is given $b^{29}$

$$
\lim _{\omega \rightarrow \infty} v_{p}=\frac{c_{0}}{\sqrt{\alpha_{\infty}}},
$$

where $c_{0}$ is the sound velocity in air. If the transfer matrix approach is considered, the equivalent wave number can be expressed with the help of the appropriate transfer matrix component (Chap. 11, p. 245 of Ref. 30):

$$
k_{e q}=\frac{1}{L} \cos ^{-1} M_{11}
$$

where $L$ is the thickness of the sample and $M_{11}$ is a component of the equivalent transfer matrix of the sample:

$$
\begin{aligned}
{[M] } & =\left[\begin{array}{ll}
M_{11} & M_{12} \\
M_{21} & M_{22}
\end{array}\right] \\
& =\left[\begin{array}{cc}
\cos \left(k_{e q} L\right) & j Z_{e q} \sin \left(k_{e q} L\right) \\
\frac{j}{Z_{e q}} \sin \left(k_{e q} L\right) & \cos \left(k_{e q} L\right)
\end{array}\right],
\end{aligned}
$$

with $Z_{e q}$ the characteristic impedance of the medium.

In Fig. 1, the theoretical phase velocity (given by JCA model) of a melamine foam is plotted versus frequency in a $\log -\log$ plot where the HF asymptote is defined by the horizontal straight line at the value $c_{0} / \sqrt{\alpha_{\infty}}$. This asymptote does not depend on frequency. The LF behavior can also be

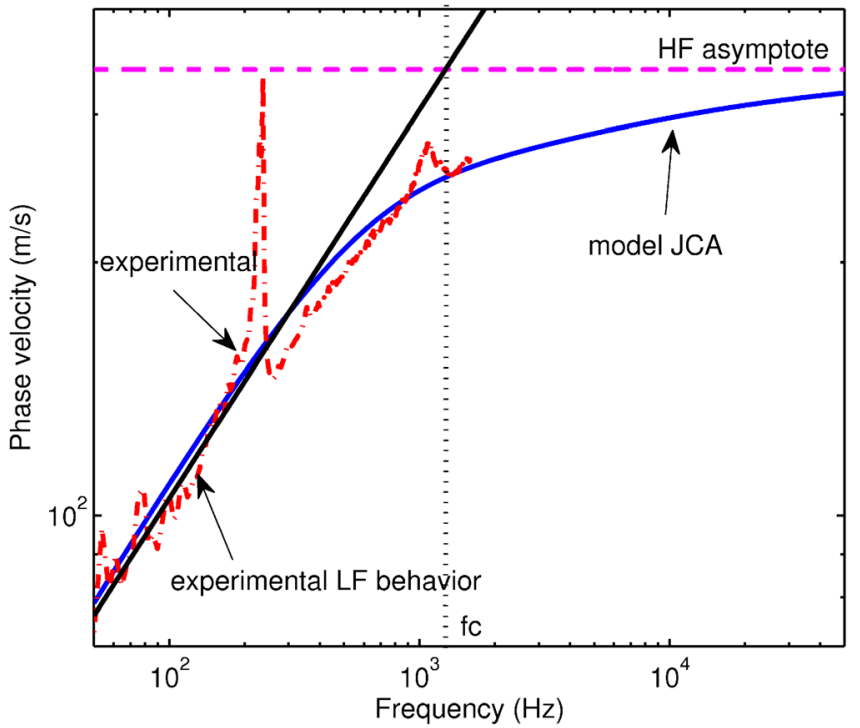

FIG. 1. Experimental phase velocity versus frequency with the experimental low frequency (LF) behavior and the high frequency (HF) asymptote for the melamine foam.

represented by a straight line. The intersection between the LF behavior and HF asymptote gives Biot's characteristic frequency $f_{c}$. A measurement of this frequency can provide the Biot porosity [with help of Eq. (4)].

\section{Remarks}

(1) $f_{c}$ depends linearly on $\beta / \sqrt{\alpha_{\infty}}$ with $\beta=\phi_{B} / \sqrt{\alpha_{\infty}}$. If $\beta$ is small, the experimental determination of the low frequency behavior can be a difficult task. Therefore this method is well adapted for highly porous materials with values of $\beta$ close to one.

(2) This method could be used to determine the static flow resistivity of a porous in case Biot porosity is known.

\section{B. Experimental details}

For determining the Biot porosity from the low frequency method, Eq. (4) assumes that the static airflow resistivity and tortuosity must first be known. In this study, the static airflow resistivity is measured using the method proposed by Stinson and Daigles, ${ }^{31}$ and the tortuosity by the ultrasound method. ${ }^{32,33}$ Moreover, if the DE porosity needs to be deduced from Eq. (2), the open porosity must also be known. Here, it is measured by the weight differential approach. ${ }^{15}$ For eventual acoustic simulations using the JCA model, the viscous and thermal characteristic lengths (respectively, $\Lambda$ and $\Lambda^{\prime}$ ) can be obtained by ultrasonic measurements. ${ }^{32,33}$ Note that these latter two properties are not required for determining the Biot and DE porosities. In the following, each material parameter will be defined by a mean value $\bar{x}$ and a standard deviation $s$, such that $x=\bar{x} \pm s$.

Concerning the phase velocity measurement, the transfer matrix of the sample can be determined with a technique developed recently by Salissou et al., the three-microphone and two-load method. ${ }^{34}$ To improve the measurements at low frequencies, it is recommended to use a large diameter impedance tube (with a tube diameter of $100 \mathrm{~mm}$ ). This 
TABLE I. Johnson-Champoux-Allard (JCA) parameters of the studied porous samples measured with conventional methods (mean \pm standard deviation).

\begin{tabular}{|c|c|c|c|c|c|}
\hline & $\Lambda(\mu \mathrm{m})$ & $\Lambda^{\prime}(\mu \mathrm{m})$ & $\alpha_{\infty} \pm 0.05$ & $\sigma\left(\mathrm{Pa} . \mathrm{s} / \mathrm{m}^{2}\right) \pm 300$ & $\phi_{O}(\%) \pm 3$ \\
\hline Melamine foam & $90 \pm 5$ & $180 \pm 10$ & 1.03 & 9958 & 98.2 \\
\hline Felt & Not used & Not used & 1.29 & 29800 & 98.6 \\
\hline Polyurethane foam & Not used & Not used & 1.89 & 17840 & 98.2 \\
\hline Aluminum foam with DE pores & $101 \pm 4$ & $352 \pm 14$ & 2.25 & 19713 & 64.5 \\
\hline
\end{tabular}

three-microphone technique is used to measure the normal sound absorption coefficient and sound transmission loss of the sample coupled to an air cavity and a rigid termination. The frequency range was chosen between 50 and $1600 \mathrm{~Hz}$ to make sure that only plane waves exist in the tube. With the help of two microphones in front of the sample and a third microphone localized on the hard wall backing, it is possible to measure the transfer matrix and deduce the transmission loss. The sound pressure excitation is random noise in linear regime. The majority of repeatability errors come from the way the sample is positioned in the tube: Special attention was therefore paid to this positioning.

\section{Validation of the method on samples without DE pores}

Results on different samples (without DE pores) are presented in this section. The experimental procedure is simple. First, static resistivity and tortuosity are measured as discussed previously. Second, the phase velocity is deduced from Eqs. (5) and (7), where transfer matrix coefficient $M_{11}$ is measured using the three-microphone and two-load method. Then the phase velocity is plotted versus frequency together with its LF and HF behaviors (in log-log plot). The LF behavior is obtained from the linear curve fitting on the phase velocity at low frequencies. The HF behavior is given by the horizontal line defined in Eq. (6). Third, the characteristic frequency $f_{c}$ is found at the intersection of the LF and HF lines. Because of the low frequency limit of the used impedance tube, only samples for which $f_{c}$ is greater than $200 \mathrm{~Hz}$ are measured. Finally, once $f_{c}, \sigma$, and $\alpha_{\infty}$ are known, Eq. (4) is used to deduce the Biot porosity $\phi_{B}$.

The samples studied in this section are those without DE pores $\left(\phi_{O}=\phi_{B}\right)$ and with a measurable open porosity to validate the present approach. The first sample is a melamine foam of thickness $L=50 \mathrm{~mm}$. Its JCA parameters are measured by conventional methods (see Sec. II B) and reported in Table I. The sample was pierced with nails to reduce the influence of its structural resonances during the impedance tube measurements; ${ }^{21}$ this improved the experimental low frequency determination of the phase velocity. Figure 1 presents the experimental results on the phase velocity versus frequency in a log-log plot for the melamine foam. The theoretical prediction with the JCA model is also plotted. The prediction used the material parameters given in Table I. The low and high frequency asymptotic behaviors are represented by straight lines. The intersection between these two lines occurs at the characteristic frequency. Following the proposed low-frequency method, the deduced characteristic frequency $f_{c}$ and Biot porosity $\phi_{B}$ of the melamine foam is presented in Table II. As expected for this foam without DE pores, $\phi_{B}$ is statistically equal to the open porosity $\phi_{O}$ obtained from the conventional method.

Similar measurements were also performed for two other porous materials with higher tortuosity values: One resistive felt of thickness $L=16 \mathrm{~mm}$ and one partially reticulated polyurethane foam of thickness $L=25 \mathrm{~mm}$. Their material properties are given in Table I, their phase velocities are shown in Fig. 2, and the results deduced from Fig. 2 and the present low-frequency method are given in Table II. Again, the comparison between $\phi_{B}$ measured by the present low-frequency method and $\phi_{O}$ measured by the conventional method is good.

The previous values found for the Biot porosities are given by a mean value and a standard deviation $\phi_{B}=\bar{\phi}_{B} \pm s_{\phi_{B}}$. This standard deviation represents the experimental error, which includes the repeatability errors, the error introduced by the calculation ranges in the fitting process, and the errors on the experimental determination of $\sigma$ and $\alpha_{\infty}$ (the errors are represented by the standard deviations $\left.s_{f_{c}}, s_{\sigma}, s_{\alpha_{\infty}}\right)$. The global standard deviation $s_{\phi_{B}}$ of $\phi_{B}$ is given by

$$
s_{\phi_{B}}=\sqrt{\left(\frac{\partial \phi_{B}}{\partial f_{c}} s_{f_{c}}\right)^{2}+\left(\frac{\partial \phi_{B}}{\partial \sigma} s_{\sigma}\right)^{2}+\left(\frac{\partial \phi_{B}}{\partial \alpha_{\infty}} s_{\alpha_{\infty}}\right)^{2}} .
$$

It is worth noting that the choice of frequency bandwidth for the fitting process had important consequences on the experimental error and for minimizing the error introduced by the low frequency fitting process of the phase velocity, the frequency range around a structural resonance peak was removed from the calculation for all samples.

The results presented here come from one sample of each material. Other samples of the same materials were tested, and very similar results were found.

For polyurethane foam, a Biot porosity mean value is found higher than $100 \%$. Of course this porosity value has no physical meaning. It is an experimental result that is within the $4 \%$ experimental error from the true porosity value.

TABLE II. Material parameters of the studied porous samples deduced from the present low-frequency method (mean \pm standard deviation).

\begin{tabular}{lrrc}
\hline \hline & \multicolumn{1}{c}{$f_{c}(\mathrm{~Hz})$} & \multicolumn{1}{c}{$\phi_{B}(\%)$} & $\phi_{D E}(\%)$ \\
\hline Melamine foam & $1252 \pm 45$ & $98.0 \pm 7.1$ & - \\
Felt & $3023 \pm 51$ & $99 \pm 5.2$ & - \\
Polyurethane foam & $1271 \pm 38$ & $100.3 \pm 4.3$ & - \\
Aluminum foam with DE pores & $650 \pm 34$ & $55.8 \pm 3.2$ & $8.7 \pm 3.2$ \\
\hline \hline
\end{tabular}



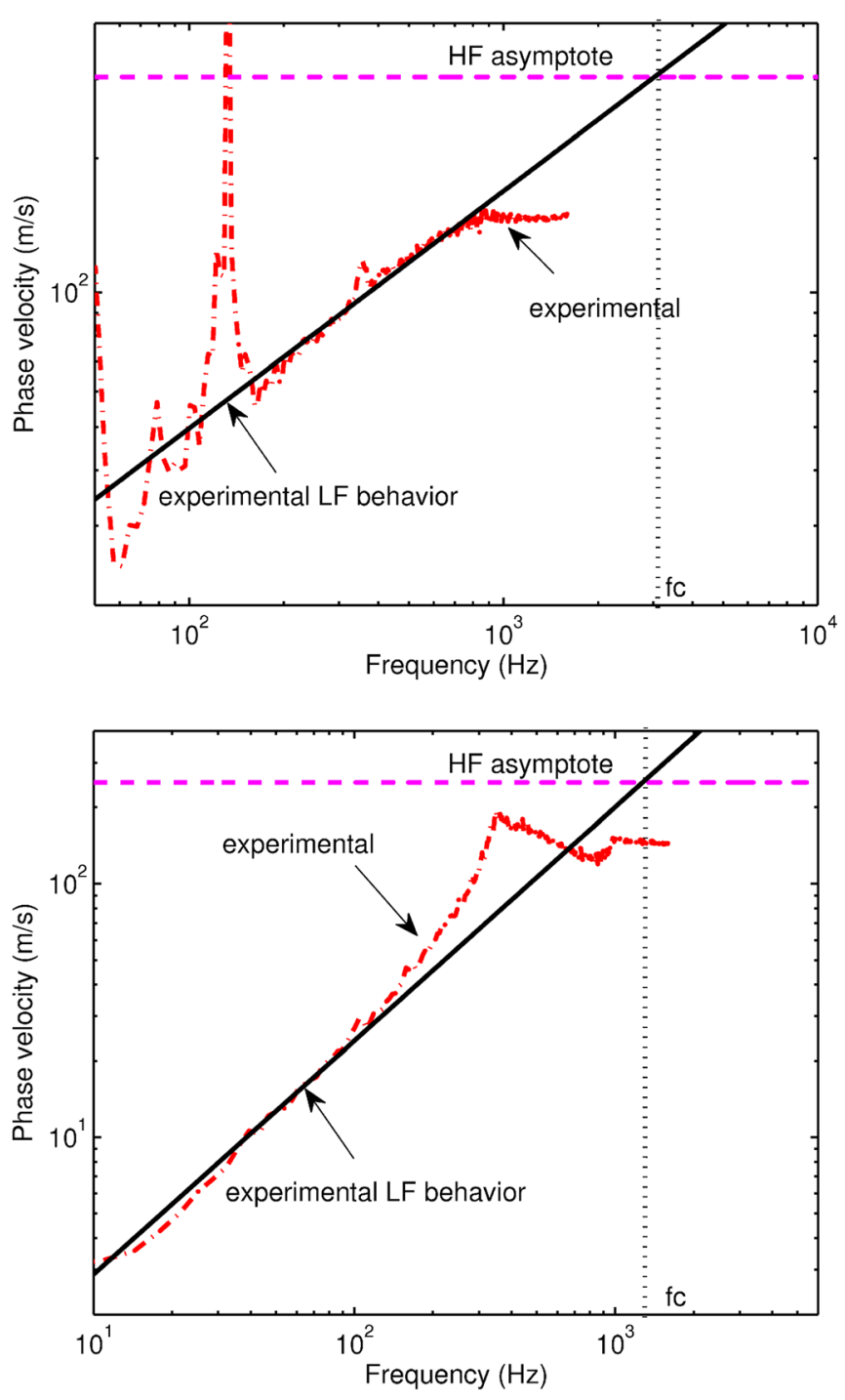

FIG. 2. (Color online) Experimental phase velocity versus frequency with the experimental low frequency (LF) behavior and the high frequency (HF) asymptote for the felt (upper figure) and the polyurethane foam (lower figure).

\section{Measurements on samples with DE pores}

The fourth sample studied is a porous aluminum (the raw material is AS7G Aluminum) for which the fabrication process is summarized in the preceding text and described precisely by Gong et al. ${ }^{9}$ The microstructure of this material suggests that it may contain DE pores. The JCA parameters are presented in Table I. To present results in higher frequencies an impedance tube with $44.4 \mathrm{~mm}$ cross section diameter is used in this part to measure the acoustic indicators of the sample. Therefore a sample with same cross section diameter is used. All JCA parameters are measured on this sample.

Figure 3 presents the experimental results on the phase velocity for the fourth sample. Note that the same sample has been used for the two porosity measurement approaches. Table II summarizes results deduced from Fig. 3 following the proposed low-frequency method. This time, one can note that the deduced Biot porosity $\left(\phi_{B}=55.8 \%\right)$ is not equal to the open porosity $\left(\phi_{O}=64.5 \%\right)$ measured with non-acoustic method. ${ }^{15}$ With respect to Eq. (2), the difference between these two porosities is the DE porosity $\left(\phi_{D E}=8.7 \%\right)$.

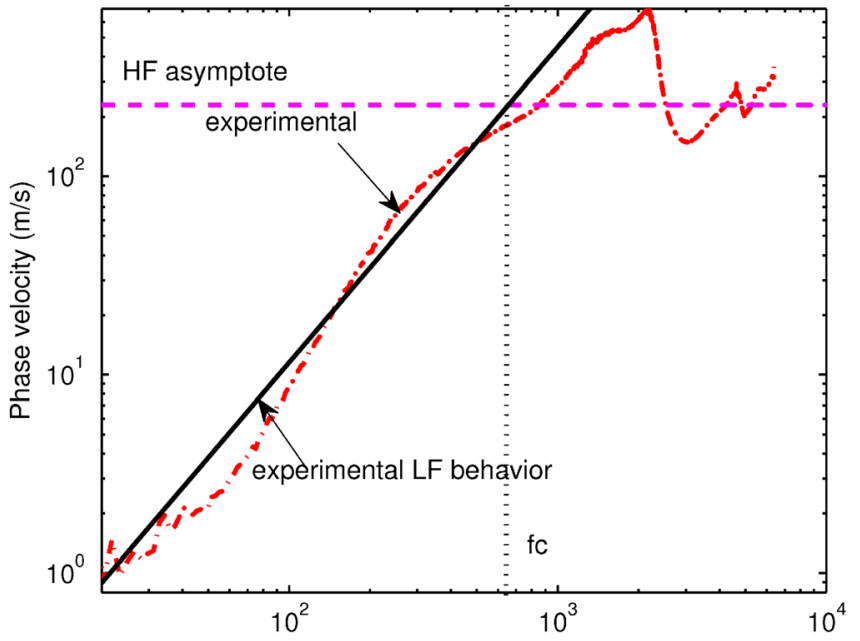

FIG. 3. (Color online) Experimental phase velocity versus frequency a porous aluminum. The plain straight line correspond the experimental LF behavior, after fitting, and the dash straight line corresponds to the theoretical HF asymptote.

To confirm the relevancy of the results, it should be noted that the value of DE porosity measured in the present paper $(8.7 \pm 3.2 \%)$ is very close to the value found with a fitting approach in the last study ${ }^{8}$ on the same sample (the value used in Ref. 8 was $7.5 \pm 3 \%$ ). Figure 4 presents the transmission loss measured on this sample. It is compared to two predictions obtained by the JCA model. In the first prediction, the five JCA parameters of Table I are used in the model (plain curve). In the second prediction, the open porosity is replaced by the Biot porosity of Table II (dot-dash curve). The experimental errors were also included in the model, and the predictions were represented with their error bars. As shown in Fig. 4, the errors on the JCA parameters introduce noticeable errors on the predictions of the sound transmission loss, which are more significant than those obtained with impedance tube measurements in the present method. To facilitate readability of the results, only the error bars on the predictions are presented in Fig. 4.

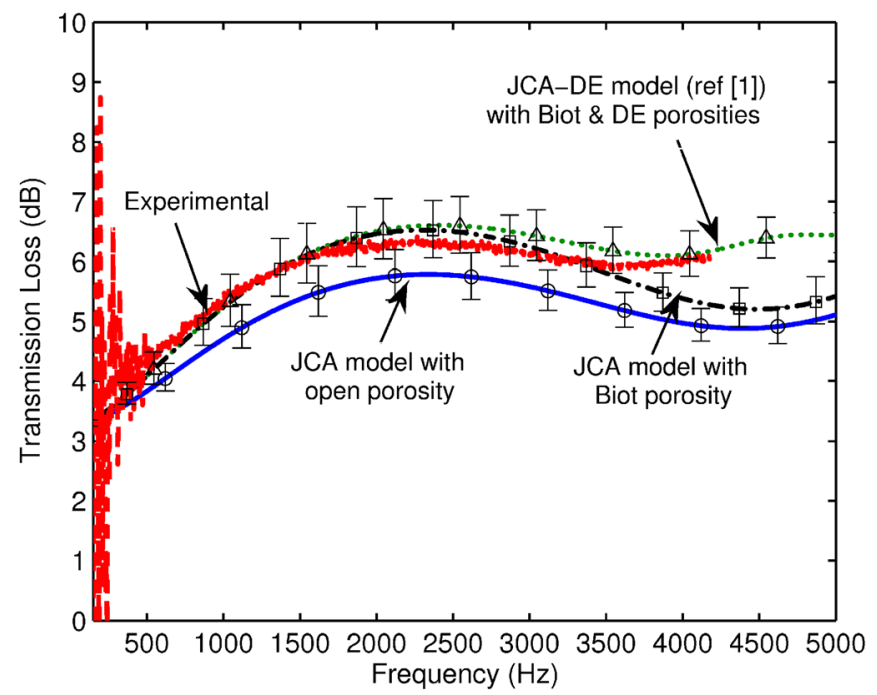

FIG. 4. Comparison between experimental results and predictions on the transmission loss for the aluminium foam sample. 
It can be observed that the JCA model fed with the open porosity does not capture the effects of DE pores, even considering error bars on the prediction. On the other hand, if the influence of the dead-end pores is accounted for by feeding the model with the Biot porosity, the result obtained from the JCA model and those of the TL measurement are in better agreement. This result confirms the presence of dead-end pores and their influence. To complete this part, Fig. 5 presents absorption coefficient of metallic foams coupled with an air cavity and a rigid wall $(50 \mathrm{~mm}$ thick air cavity).

\section{Remark}

In Figs. 4 and 5, the JCA-DE model (Ref. 8) results are shown for transmission loss and absorption coefficient. As noted in Ref. 8, in the frequency range $125-3500 \mathrm{~Hz}$, the effect of dead end consists mainly in a porosity variation, and the JCA model with Biot porosity predicts the experimental results well. Above $3500 \mathrm{~Hz}$, experimental results show that the modified JCA model with Biot porosity fails to predict the results accurately. The model proposed in Ref. 8 that both accounts for porosity correction and dead end cavity effect has to be used.

\section{E. Advantage and limitation of the low-frequency method}

The advantage of the LF method is that it can be used with relatively low measurement error for materials with high cut-off frequency $f_{c}$. It can be noticed that for a sufficiently high value of $\beta=\phi_{B} / \sqrt{\alpha_{\infty}}$, the same errors were found for low and high values of $\phi_{B}$, and the method was validated for metallic foam with DE pores. However, a limitation of the method is the existence of low frequency structural vibrations that can interfere with the measurement of phase velocity and prevent the determination of the porosities. Also, this method fails for materials with low Biot

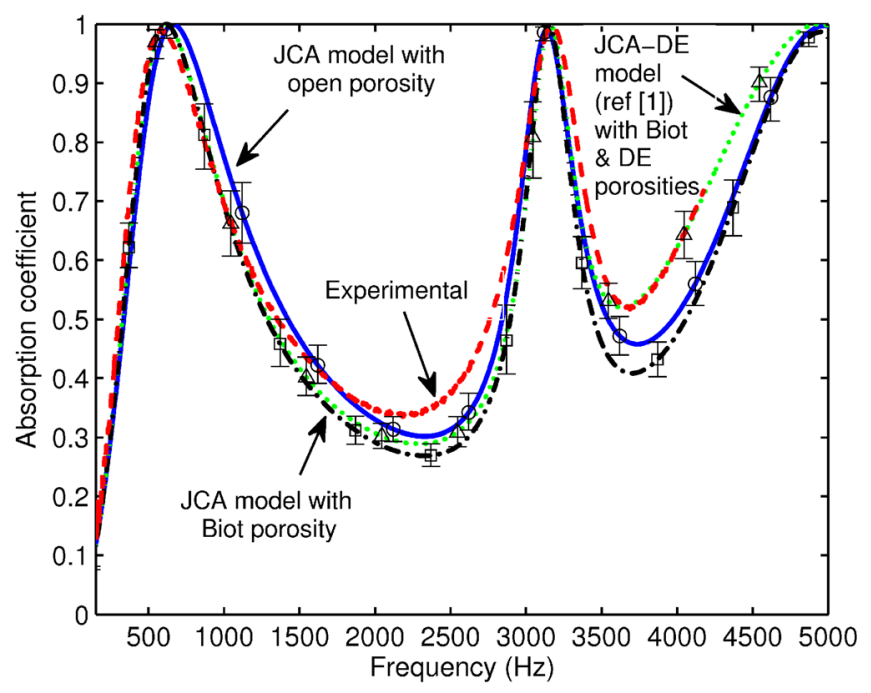

FIG. 5. Comparison between experimental results and predictions on the absorption coefficient of metallic foam coupled to an air cavity and a rigid wall $\left(l_{c a v}=50 \mathrm{~mm}\right)$. characteristic frequency $f_{c}$ (small value of $\beta$ ). For these materials, the low frequency measurement range may be too narrow for the method to be reliable. This method will be most efficient when using large cross section impedance tubes.

For materials with low values of $f_{c}$, a second acoustic method is proposed. This method involves high frequency measurements and is presented in the following section.

\section{HIGH-FREQUENCY METHOD}

\section{A. High frequency asymptotic behavior of the transmission loss}

In this method, high frequency measurements obtained from ultrasonic experiments are used in combination with the high frequency asymptotic behavior of the modulus of the logarithm of the transmission coefficient $|\ln | T(\sqrt{\omega})||$. This combination provides a straight line the intercept of which with the vertical axis at zero frequency depends on the Biot porosity. The high frequency behavior of this coefficient was studied by Moussatov et al. ${ }^{35}$ Their approach is summarized in what follows.

Assuming the porous material is homogeneous and behaves as an equivalent fluid, its sound transmission coefficient can be expressed in terms of the transfer matrix coefficients of Eq. (8) as

$$
T(\omega)=\left[\frac{2 e^{j k_{0} L}}{M_{11}+M_{22}+M_{12} / Z_{0}+M_{21} Z_{0}}\right],
$$

or

$$
\begin{aligned}
T(\omega)= & 2 e^{j k_{0} L}\left[2 \cos \left(k_{e q} L\right)+j \sin \left(k_{e q} L\right)\right. \\
& \left.\times\left(\frac{Z_{e q}}{Z_{0} \phi_{B}}+\frac{Z_{0} \phi_{B}}{Z_{e q}}\right)\right]^{-1},
\end{aligned}
$$

with

$$
k_{e q}(\omega)=\omega \sqrt{\frac{\rho_{e q}(\omega)}{K_{e q}(\omega)}}, \quad Z_{e q}(\omega)=\sqrt{K_{e q}(\omega) \rho_{e q}(\omega)},
$$

where $\rho_{e q}$ and $K_{e q}$ are, respectively, the dynamic density and Bulk modulus of the equivalent fluid porous material, $k_{0}$ is the wave number in air.

Moussatov et al. ${ }^{35}$ proposed to use three high frequency approximations. First, when the sample thickness is large compared to the length of the excitation signal, internal reflection inside the material is weak and the approximation is given by

$$
\lim _{L \gg \lambda}|T(\omega)|=\left|\frac{4 \phi_{B}}{Z_{e q} Z_{0}}\left[\frac{1}{Z_{0}}+\frac{\phi_{B}}{Z_{e q}}\right]^{-2} e^{-j k_{e q} L}\right| .
$$

The other two approximations are those, respectively, given by Johnson et al. ${ }^{29}$ and Champoux and Allard ${ }^{1}$ on the dynamic density and Bulk modulus: 


$$
\begin{aligned}
& \left.\lim _{\omega \rightarrow \infty} \rho_{e q}(\omega)=\rho_{0} \quad 1+(1-j) \frac{1}{\Lambda}\left(\frac{2 \eta}{\rho_{0} \omega}\right)^{1 / 2}\right) \\
& \lim _{\omega \rightarrow \infty} K_{e q}(\omega)=K_{a} /\left(1+(\gamma-j)(1-j) \frac{1}{\Lambda^{\prime}}\left(\frac{2 \eta}{\rho_{0} \omega \operatorname{Pr}}\right)^{1 / 2}\right) .
\end{aligned}
$$

With these asymptotic expressions, Moussatov ${ }^{35}$ proposed the HF asymptotic approximation of the transmission coefficient:

$$
\lim _{\omega \rightarrow \infty}|\ln | T(\omega)||=\ln (\varepsilon)+\sqrt{\omega}\left(\sqrt{\frac{\eta \alpha_{\infty}}{2 \gamma P_{0}}} \frac{L}{L_{e q}}\right),
$$

with parameter $\varepsilon$ and equivalent length $L_{e q}$ given by

$$
\begin{gathered}
\varepsilon=\frac{\left(1+\phi_{B} / \sqrt{\alpha_{\infty}}\right)^{2}}{4 \phi_{B} / \sqrt{\alpha_{\infty}}}, \\
L_{e q}=\left(\frac{1}{\Lambda}+\frac{\gamma-1}{\sqrt{\operatorname{Pr}} \Lambda^{\prime}}\right)^{-1},
\end{gathered}
$$

where $\eta$ is the dynamic viscosity of air, $\gamma$ is the specific heat ratio, $P_{0}$ is the atmospheric static pressure and $\operatorname{Pr}$ is the Prandtl number.

In the high frequency domain, the logarithm varies linearly with the square roots of angular frequency, and expression (16) can be rewritten as

$$
\lim _{\omega \rightarrow \infty}|\ln | T(\omega)||=A+\sqrt{\omega} B
$$

with $A=\ln (\varepsilon)$. When plotting $|\ln | T||$ as a function of the square root of angular frequency, $A$ represents the intersection of the HF asymptote with the vertical axis. With help of $A$ value, Eq. (17) is solved and two roots for $\phi_{B}$ are found:

$$
\phi_{B}=\sqrt{\alpha_{\infty}}\lfloor(2 \varepsilon-1) \pm 2 \sqrt{\varepsilon(\varepsilon-1)}\rfloor,
$$

with $\varepsilon=e^{A}$. by

For physical reasons the only acceptable root is given

$$
\phi_{B}=\sqrt{\alpha_{\infty}}\lfloor(2 \varepsilon-1)-2 \sqrt{\varepsilon(\varepsilon-1)}\rfloor .
$$

\section{Remark}

Figure 6 shows the root $\beta=\phi_{B} / \sqrt{\alpha_{\infty}}$ plotted as a function of $A=\ln (\varepsilon)$. As noted in previous studies, ${ }^{24,35}$ for materials with $\beta=\phi_{B} / \sqrt{\alpha_{\infty}} \approx 1$, the error on $A$ gives a large error on $\beta$. This shows the weak dependence of the transmission coefficient with porosity for $\beta$ close to one. The smaller the value of $\beta$, the weaker the error on $\phi_{B}$ (this error is induced by the determination of $A$ on the vertical $|\ln | T||$ axis). This HF method is well adapted for materials with small value of $\beta$.

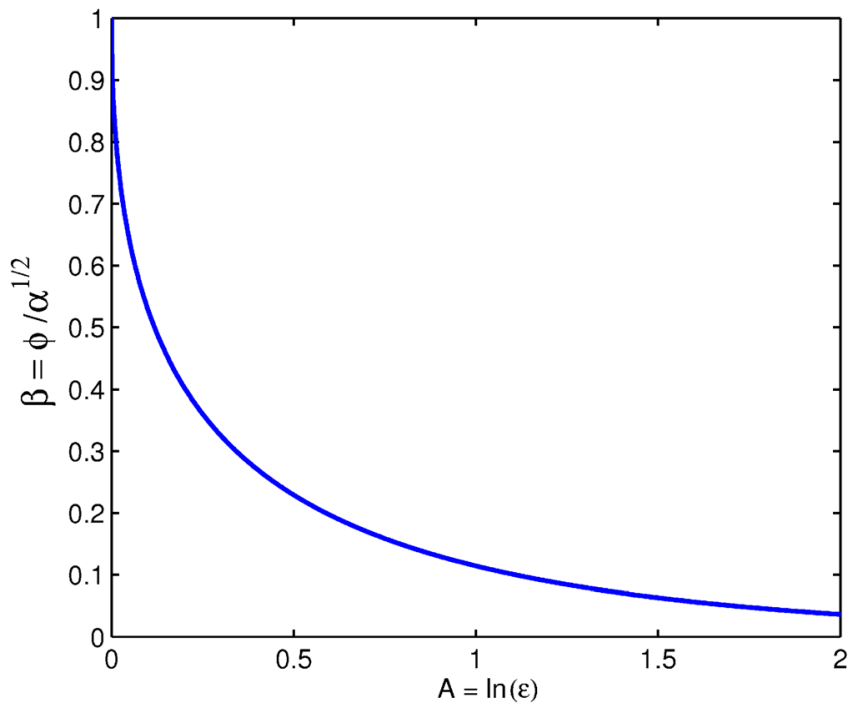

FIG. 6. (Color online) Simulation of root $\beta=\phi_{B} / \sqrt{\alpha_{\infty}}$ versus $A=\ln (\varepsilon)$.

\section{Remark}

The HF slope $s$ of the function $f(\sqrt{\omega})$ $=\lim _{\omega \rightarrow \infty}|\ln | T(\sqrt{\omega})||$ allows the measurement the measurement of Leq if $\alpha_{\infty}$ is known by $L_{e q}$ $=\left(\sqrt{\left(\eta \alpha_{\infty} / 2 \gamma P_{0}\right)}(L / s)\right)$ or the measurement of $\alpha_{\infty}$ and $L_{e q}$ with used of two different pressures (see Moussatov et al. ${ }^{35}$ ).

\section{B. Experimental setup}

Two pairs of air-coupled piezoelectric transducers, Ultran NCG50-D25 (central frequency of $50 \mathrm{kHz}$ ), NCG200D25 (central frequency of $200 \mathrm{kHz}$ ) were used. The transducers active diameter is $25 \mathrm{~mm}$. Two measurements are required to determine the transmission factor, one with material [receiver $i$ signal $s_{i}(t)$ ] and one without material [receiver $i$ signal $\left.y_{i}(t)\right]$. The fast Fourier transform of these two signals are calculated, $Y_{i}(\omega)=\operatorname{FFT}\left(y_{i}(\mathrm{t})\right)$ and $S_{i}(\omega)=\operatorname{FFT}\left(s_{i}(\mathrm{t})\right)$. The transmission coefficient for each frequency is given by the ratio of the receiver signal between the configurations with and without material:

$$
T(\omega)=\frac{S(\omega)}{Y(\omega)} .
$$

The method of resonant peak minus $3 \mathrm{~dB}$ described by Groby et $a l .{ }^{26}$ has been chosen, where only the information
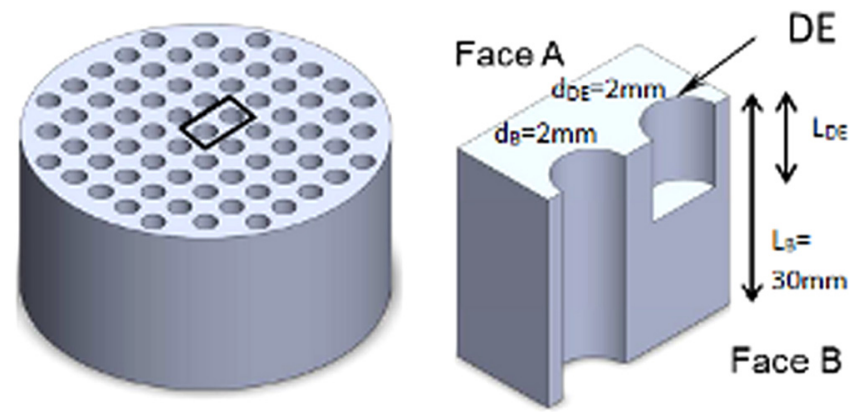

FIG. 7. Scheme of the simplified samples with surface DE pores. 
close to the transducer resonant peak is considered for the post treatment. For each double transducer, a pulsation bandwidth is defined: $\Delta \omega_{i}=\left[\omega_{i-}, \omega_{i+}\right]$. The measured HF transmission coefficient is defined on the two bandwidths:

$$
T(\omega)=\left[T_{\Delta \omega 1}(\omega) \quad T_{\Delta \omega 2}(\omega)\right]
$$

with $T_{\Delta \omega i}(\omega)=\left(S_{i}(\omega) / Y_{i}(\omega)\right)_{\Delta \omega i}$.

On the limited frequency bandwidth, the linear fitting is used to find the two constants $A$ and $B$ of the straight line [Eq. (19)]. A linear fitting is applied to determine $A$ and $B$. Special attention has to be paid to the position of the detector. Indeed, the porosity measurement is highly dependent on the error due to the detector position. The transducers are not focalized and the sample is positioned very close to the transmitter transducer $(10 \mathrm{~mm})$.

Similar to the LF method, the experimental Biot porosity is given by a mean value and a standard deviation. This standard deviation represents the experimental error, which includes the repeatability errors and the errors on the experimental determination of $\alpha_{\infty}$.

\section{Validation on simplified samples with surface DE pores}

The samples presented in this section are simplified samples with surface DE pores. The samples are perforated solid layers as depicted in Fig. 7. They are perforated by straight cylindrical pores fully open on both ends to the surrounding medium (the Biot pores). Also, semi-open pores are drilled on its surfaces (the DE pores). The thickness of both samples is $30 \mathrm{~mm}$, the length of their DE pores varies between 23 and $27 \mathrm{~mm}$, and the diameter of the perforations (Biot and DE pores) is $2 \mathrm{~mm}$. Even if the central frequencies of the transducers are high and the wavelengths are close to the hole sizes, it is supposed here that for these frequencies, the scattering geometry effects are irrelevant for this sample (material with simple geometries incorporating uniform straight cylindrical pores). Indeed, the medium is supposedly ordered for this kind of sample. The sample diameter is $29 \mathrm{~mm}$, but the active diameter of the transducers is $25 \mathrm{~mm}$, and then when the transducer is positioned at the center and very close to the sample (more than $10 \mathrm{~mm}$ ), only the perforations localized on the internal perforation circle have to be considered. Table III summarizes the configurations of the two samples. Each sample is tested on both faces, and the value of their Biot porosity is determined with the proposed HF method determined (with consideration for the measurement errors). The theoretical values (reference values) of porosities (open, DE and Biot porosities) are summarized in Table III. Each sample was tested at least 10 times (tested on both faces).

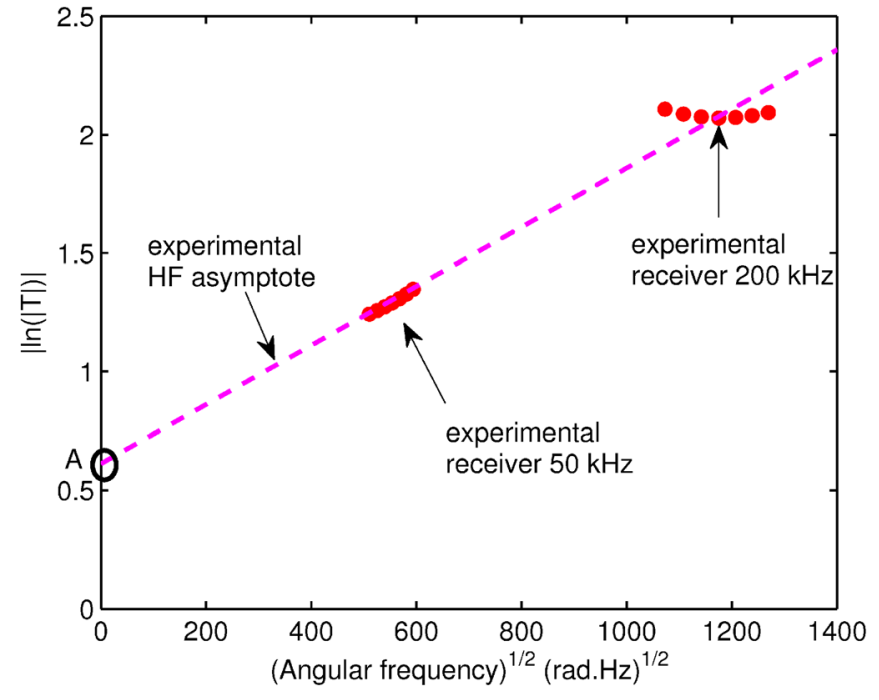

FIG. 8. Logarithm of the modulus of the transmission coefficient of simplified Sample 2 with surface DE pores. The experimental results found with two pairs of air-coupled piezoelectric transducers are represented by the dots. The experimental HF asymptote (linear fitting of exper-imental results) is represented by the dashed curve.

Figure 8 shows the results obtained for the simplified sample of Fig. 6. The experimental $|\ln | T(\sqrt{\omega})||$ measured with two pairs of air-coupled piezoelectric transducers and the HF asymptote (found with a linear fitting) is plotted.

\section{Remark}

The ultrasonic reflection measurement method ${ }^{24}$ (multi incidence angle methods) has been tested with these kind of simplified samples in a configuration where the face showing all the pores (face A) is on the source side. As expected, incorrect values were obtained, showing that this method cannot be used for this type of samples with surface DE pores. In fact, the values obtained correspond to those that would have been found if all the perforations (the dead-ends included) were fully opened on both side through the layer. The method using reflections is based on the assumption that the surface porosity is equivalent to the volume porosity. This can be the case for statistically isotropic materials but not for our samples.

\section{Validation on a simplified sample with internal DE pores}

The sample presented in this section is a simplified sample with internal DE pores as shown in Fig. 8. Three identical samples were made by three-dimensional (3D) printing. Each sample contains 49 straight cylindrical pores fully

TABLE III. Characteristics of simplified samples with surface DE pores; the sample has DE pores with different $L_{D E}$. The theoretical (reference) and measured porosities for the two simplified samples with surface DE pores (mean \pm standard deviation) are presented.

\begin{tabular}{|c|c|c|c|c|c|c|c|}
\hline & Number of Biot hole & Number of DE hole & $L_{D E}$ & $\phi_{O}(\%)$ reference & $\phi_{D E}(\%)$ reference & $\phi_{B}(\%)$ reference & $\phi_{B}(\%)$ Proposed HF method \\
\hline Sample 1 & 21 & $1,16,15$ & $24,25,26$ & 35.93 & 22.49 & 13.44 & $11.05 \pm 3.76$ \\
\hline Sample 2 & 32 & 18 & 25 & 30.08 & 9.60 & 20.48 & $20.35 \pm 2.5$ \\
\hline
\end{tabular}




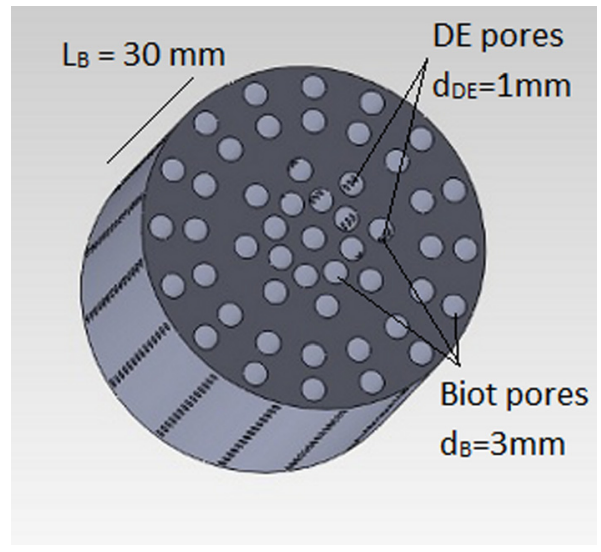

FIG. 9. Scheme of the simplified samples with internal DE pores. Note that before testing, the exterior end terminations of the lateral pores are plugged.

opened on both ends (the Biot pores) and 224 semi-open lateral pores (the DE pores). Half of the lateral pores are 11$\mathrm{mm}$ long and connected to the eight Biot pores distributed on the internal perforation circle. The other half of the lateral pores are 15-mm thick and connected to the eight Biot pores distributed on the second internal perforation circle (Fig. 9). The diameter of the Biot pores is $3 \mathrm{~mm}$, and the diameter of the DE pores is $1 \mathrm{~mm}$. As noted in the preceding text, it is supposed here that for these frequencies, the scattering geometry effects are irrelevant for this sample. The sample thickness is $30 \mathrm{~mm}$, the sample diameter is $44 \mathrm{~mm}$, but the active diameter of the transducers is $25 \mathrm{~mm}$; only the perforations localized on the internal perforation circle have to be considered. The theoretical values (reference values) of all porosities are summarized in Table IV.

Figure 10 shows the experimental $|\ln | T(\sqrt{\omega})||$ measured on one simplified sample and its HF asymptote (found with a linear regression). This measurement is repeated five times for each three printed samples (total of 15 measurements). With these measurements, the Biot porosity is deduced from the present HF method and its statistics reported in Table IV. For this sample, additional measurements with $100 \mathrm{kHz}$ transducers (Ultran NCG100-D25) are presented here to improve the validation of the present approach on a sample with internal DE pores. The comparison between the reference value of the Biot porosity and the measured value are acceptable, despite of the measurement error that is relatively high.

\section{E. Advantage and limitation of the high-frequency method}

Relatively low errors for low $\beta=\phi_{B} / \sqrt{\alpha_{\infty}}$ were found. The method was validated for simplified samples with surface DE pores and interior DE pores. The method uses the same experimental configuration to measure $L_{e q}$ and $\alpha_{\infty}$ as

TABLE IV. Theoretical (reference) and measured porosities for the simplified sample with internal DE pores (mean \pm standard deviation).

\begin{tabular}{lccc}
\hline \hline $\begin{array}{l}\phi_{O}(\%) \\
\text { reference }\end{array}$ & $\begin{array}{l}\phi_{D E}(\%) \\
\text { reference }\end{array}$ & $\begin{array}{c}\phi_{B}(\%) \\
\text { reference }\end{array}$ & $\begin{array}{c}\phi_{B}(\%) \\
\text { Proposed HF method }\end{array}$ \\
\hline 49.48 & 22.06 & 27.42 & $27.18 \pm 3.26$ \\
\hline \hline
\end{tabular}

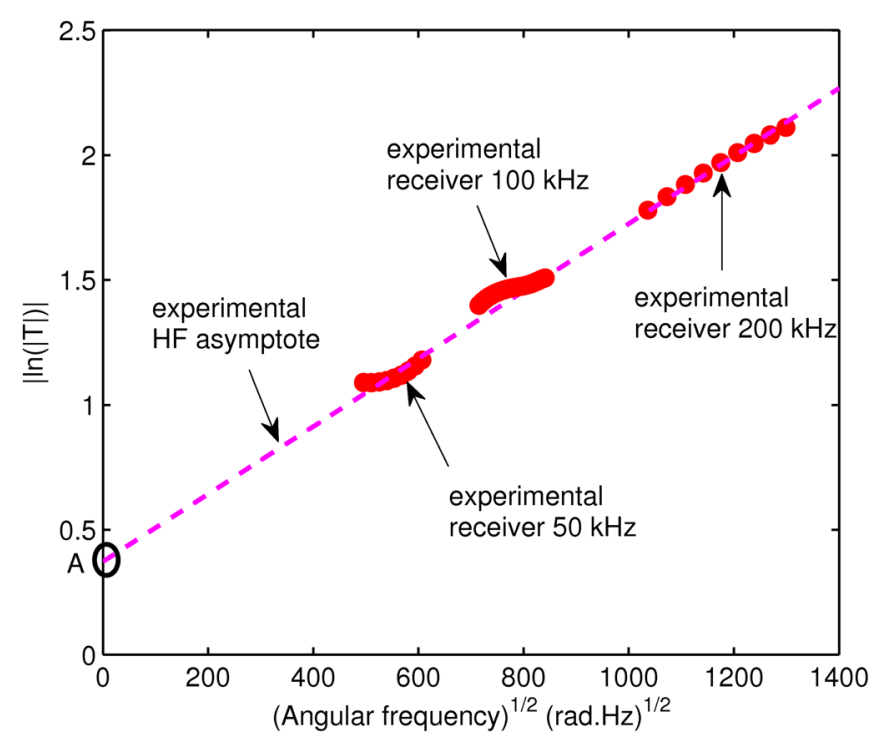

FIG. 10. Logarithm of modulus of the transmission coefficient for one simplified sample with interior DE pores. The experimental results found with three pairs of air-coupled piezoelectric transducers are represented by the dots. The experimental HF asymptote (linear fitting of experimental results) is represented by the dashed curve.

in references. ${ }^{26,32}$ However, high errors were found for value $\beta=\phi_{B} / \sqrt{\alpha_{\infty}}$ greater than 0.5 . For higher porosities, the low frequency method may be considered. This method is very sensitive to variations in transducers positions.

\section{CONCLUSION}

Two different experimental acoustic methods were presented for the measurement of the Biot porosity. With the knowledge of the open porosity (measured with classical methods), it is possible to deduce the dead-end porosity of materials incorporating dead-end pores. These methods are based on acoustic transmission measurements and on low and high frequencies behaviors of acoustic functions (phase velocity, transmission loss). The two methods allow the measurement of the porosity for a wide range of the parameter $\beta=\phi_{B} / \sqrt{\alpha_{\infty}}$. The high frequency method (ultrasonic method) is based on the high frequency behavior of the logarithm of the transmission coefficient, and it is well adapted for materials with low values of $\beta$. The low frequency method (transmission tube method) is based on the high (theoretical) and low (experimental) frequency behavior of the phase velocity. It is useful for materials with higher values of $\beta$. Relatively low experimental error was found, and materials containing DE pore were tested for each method. To improve the measurement quality and reduce the experimental error, it could be considered for the low frequency method to better control the structural resonance of the material and to use a larger tubes to measure very low viscous characteristic frequencies. Concerning the high frequency method, it is possible to use large bandwidth frequency transducers.

\footnotetext{
${ }^{1}$ Y. Champoux and J. F. Allard, "Dynamic tortuosity and bulk modulus in air-saturated porous media,” J. Appl. Phys. 70, 1975-1979 (1991).

${ }^{2}$ T. Bourbié, O. Coussy, and B. Zinszner, Acoustic of Porous Media (Technip, Paris, 1986), pp. 339.
} 
${ }^{3}$ I. Fatt, "Influence of dead end pores on relative permeability of porous media," Science 134, 1750-1751 (1961).

${ }^{4}$ J. Hrabe, S. Hrabetova, and K. Segeth, "A model of effective diffusion and tortuosity in the extracellular space of the brain," Biophys. J. 87, 1606-1617 (2004).

${ }^{5}$ F. Chevillotte, C. Perrot, and R. Panneton, "Microstructure based model for sound absorption predictions of perforated closed-cell metallic foams," J. Acoust. Soc. Am. 128(4), 1766-1776 (2010).

${ }^{6}$ N. Fang, D. J. Xi, J. Y. Xu, M. Ambati, W. Srituravanich, C. Sun, and X. Zhang, "Ultrasonic metamaterials with negative modulus," Nat. Mater. 5, $452-456$ (2006).

${ }^{7}$ S. H. Lee, C. M. Park, Y. M. Seo, Z. G. Wang, and C. K. Kim, "Metamaterial with negative modulus," J. Phys. Acoustic Condens. Matter 21, 175704 (2009).

${ }^{8}$ T. Dupont, P. Leclaire, O. Sicot, X. L. Gong, and R. Panneton, "Acoustic properties of air-saturated porous materials containing dead-end porosity," J. App. Phys. 110, 094903 (2011).

${ }^{9}$ X. L. Gong, Y. Liu, S. Y. He, and J. Lu, "Manufacturing and low-velocity impact response of a new composite material, metal porous polymer composite," J. Mater. Sci. Technol. 20, 65-68 (2004).

${ }^{10}$ L. L. Beranek, "Acoustic impedance of porous materials," J. Acoust. Soc. Am. 13, 248-260 (1942).

${ }^{11}$ ASTM D2856-94, Standard Test Method for Open-Cell Content of Rigid Cellular Plastics by the Air Pycnometer (ASTM, West Conshohocken, PA, 1994), pp. 143-148.

${ }^{12}$ P. Leclaire, O. Umnova, K. V. Horoshenkov, and L. Maillet, "Porosity measurement by comparison of air-volumes," Rev. Sci. Inst. 74, 13661370 (2003).

${ }^{13}$ Y. Champoux, M. R. Stinson, and G. A. Daigle, "Air-based system for the measurement of the porosity," J. Acoust. Soc. Am. 89, 910-916 (1990).

${ }^{14}$ R. Panneton and E. Gros, "A missing mass method to measure the open porosity of porous solids," Acta Acust. Acust. 91, 342-348 (2005).

${ }^{15}$ Y. Salissou and R. Panneton, "Pressure/mass method to measure the open porosity of porous solids," J. App. Phys. 101, 124913 (2007).

${ }^{16}$ P. Leclaire, M. J. Swift, and K. V. Horoshenkov, "Determining the specific area of porous acoustic material from water extraction data," J. App. Phys. 84(12), 6886-6890 (1998).

${ }^{17}$ P. Gibb, M. J. Barcelona, J. D. Ritchey, and M. H. LeFaivre, "Effective porosity of geologic material-First annual report," Report for State Water Survey Division, ENR, Illinois Dept., 1984.

${ }^{18}$ R. A. Ketcham and G. J. Iturrino, "Nondestructive high-resolution visualization and measurement of anisotropic effective porosity in complex lithologies using high-resolution X-ray computed tomography," J. Hydrol. 302, 92-106 (2005).

${ }^{19}$ H. Taud, R. Martinez-Angeles, J. F. Parrot, and L. Hernandez-Escobedo, "Porosity estimation method by X-ray computed tomography," J. Sci. Eng. 47, 209-217 (2005)

${ }^{20} \mathrm{R}$. Panneton and X. Olny, "Acoustical determination of the parameters governing viscous dissipation in porous media," J. Acoust. Soc. Am. 119(4), 2027-2040 (2006).
${ }^{21} \mathrm{X}$. Olny and R. Panneton, "Acoustical determination of the parameters governing thermal dissipation in porous media," J. Acoust. Soc. Am. 123(2), 814-824 (2008).

${ }^{22}$ O. Doutres, Y. Salissou, N. Atalla, and R. Panneton, "Evaluation of the acoustic and non-acoustic properties of sound absorbing materials using a three-microphone impedance tube," Appl. Acoust. 71, 506-509 (2010).

${ }^{23}$ Z. E. A. Fellah, M. Fellah, N. Sebaa, W. Lauriks, and C. Depollier, "Measuring flow resistivity of porous materials at low frequencies range via acoustic transmitted waves," J. Acoust. Soc. Am. 119(4), 1926-1928 (2006).

${ }^{24}$ Z. E. A. Fellah, S. Berger, W. Lauriks, C. Depollier, C. Aristegui, and J.-Y. Chapelon, "Measuring the porosity and the tortuosity of porous materials via reflected waves," J. Acoust. Soc. Am. 113(5), 2424-2433 (2003).

${ }^{25}$ O. Umnova, K. Attenborough, H. C. Shin, and A. Cummings, "Deduction of tortuosity and porosity from acoustic reflection and transmission measurements on thick samples of rigid-porous materials," Appl. Acoust. 66, 607-624 (2005).

${ }^{26}$ J.-P. Groby, E. Ogam, L. De Ryck, N. Sebaa, and W. Lauriks, “Analytical method for the ultrasonic characterization of homogeneous rigid porous materials from transmitted and reflected coefficients," J. Acoust. Soc. Am. 127(2), 764-772 (2010)

${ }^{27}$ T. Dupont, P. Leclaire, and R. Panneton, "Methods for measuring the porosities of porous materials incorporating dead-end pores," in Proceedings of the Symposium on the Acoustics of Poro-Elastic Materials (SAPEM), Paper Session 1-1, Ferrara, Italy (December 2011).

${ }^{28} \mathrm{M}$. A. Biot, "Theory of propagation of elastic waves in a fluid-saturated porous solid. Part II. Higher frequency range,” J. Acoust. Soc. Am. 28(2), 179-191 (1956).

${ }^{29}$ D. L. Johnson, J. Koplik, and R. Dashen, "Theory of dynamic permeability and tortuosity in fluid-saturated porous media," J. Fluid Mech. 176, 379402 (1987).

${ }^{30}$ J. F. Allard and N. Atalla, Propagation of Sound in Porous Media: Modelling Sound Absorbing Materials (Wiley and Sons, Southern Gate, Chichester, West Sussex, UK, 2009), 358 pp.

${ }^{31}$ M. R. Stinson and G. A Daigle, "Electronic system for the measurements of flow resistance," J. Acoust. Soc. Am. 83, 2422-2428 (1988).

${ }^{32}$ P. Leclaire, L. Kelders, W. Lauriks, C. Glorieux, and J. Thoen, "Determination of the viscous characteristic length in air-filled porous materials by ultrasonic attenuation measurements," J. Acoust. Soc. Am. 99, 1944-1948 (1996).

${ }^{33}$ B. Brouard, B. Castagnède, M. Henry, D. Lafarge, and S. Sahraoui, "Mesure des propriétés acoustiques des matériaux poreux (Experimental methods to measure the acoustic properties of porous material)," Techniques de L'Ingénieur R6, 120-122 (2003).

${ }^{34}$ Y. Salissou, R. Panneton, and O. Doutres, "Complement to standard method for measuring normal incidence sound transmission loss with three microphones," J. Acoust. Soc. Am. 131(3), EL216-EL222 (2012).

${ }^{35}$ A. Moussatov, C. Ayrault, and B. Castagnède, "Porous material characterization - ultrasonic method for estimation of tortuosity and characteristic length using a barometric chamber," Ultrasonics 39, 195-202 (2001). 